\title{
Evolution de la composition chimique des eaux de drainage dans un système d'épuration du lisier par le sol
}

\section{Drainage water quality changes from a soil treatment process for pig slurry}

\author{
par J. Martinez et P. Peu
}

Cemagref, Unité de Recherche Gestion des Effluents d'Elevage et des Déchets Municipaux

\begin{abstract}
The current study reports on operational and performance aspects of a land treatment facility "Solepur", for managing surplus pig slurry (986 $\left.\mathrm{m}^{3} / \mathrm{ha} / \mathrm{yr}\right)$ over a 5 -year period (1991-1995); followed by a monitoring phase on the status of the soil-water system over an additional 5-year period (1996-2000). When large applications of pig slurry are made to soil, intense microbial oxidation occurs which results in the breakdown of organic matter. Soil is able to retain most of the elements applied in slurry particularly in the 0 $20 \mathrm{~cm}$ layer, $80 \%$ of the carbon, phosphorus, copper and zinc retained by the soil are located in this layer. Most of the immobilized nitrogen (70\%) also accumulates in the topsoil. A number of elements including nitrate-nitrogen, potassium, calcium, chloride and sulphate were heavily leached at rates ranging from 400 to $600 \mathrm{~kg} \mathrm{ha} \mathrm{I}^{-1} \mathrm{yr}^{-1}$.
\end{abstract}

\section{IINTRODUCTION}

Le sol est un compartiment fondamental des éco-systèmes terrestres, un support physique et nutritif pour les cultures, un réservoir et un filtre essentiels au cycle de l'eau. Il assure toutes ces fonctions par le jeu complexe d'interactions multiples entre ses constituants, vivants et inanimés. La qualité de l'eau qui migre vers les aquifères dépend des capacités du sol et du sous-sol qu'elle traverse à éliminer les matières polluantes qu'elle contient. Les mécanismes qui permettent de transformer une eau de surface, plus ou moins chargée en matières en suspension et en éléments organiques ou minéraux dissous, en une eau potable, constituent les propriétés épuratrices du sol [1].

L'homme a utilisé de longue date ce pouvoir épurateur du sol pour régénérer la qualité des eaux qu'il a souillées. L'épandage utilisé comme procédé d'épuration permet de traiter les effluents susceptibles d'être dégradés biologiquement dans le sol, qu'il s'agisse d'eaux résiduaires ou de déchets solides, d'origine urbaine ou agricole. Cependant, on ne pourra parler de résorption quasi complète de la pollution grâce à l'épandage, qu'à deux conditions [3] :

- le transfert de la charge polluante ne concerne que des éléments ne conduisant pas à une pollution du milieu récepteur (soit de par leur nature, soit parce que leur concentration restera faible),
- il n'y a pas d'accumulation dans le sol d'éléments pouvant condamner à terme toute spéculation agricole.

C'est en partie sur la base de ces considérations théoriques qu'est apparue l'idée d'aménager une parcelle «sacrifiée " pour évaluer en vraie grandeur les performances d'un procédé de traitement du lisier de porc fondé sur la capacité intrinsèque du sol à l'épuration de l'azote des lisiers par le jeu de processus de nitrification-dénitrification [4].

Une opération baptisée "SOLEPUR » fut ainsi mise en place en 1990 basée sur l'aménagement d'une parcelle épuratrice en drainage intégral (macro-lysimètre), destinée à purifier des doses massives et répétées de lisier de porc brut, un système de bassins permettant la collecte, le stockage et un complément de traitement des percolats recueillis (dénitrification) et une parcelle d'épuration finale recevant par irrigation l'effluent dénitrifié. La parcelle traitée a reçu des doses massives et contrôlées de lisier de porc $\left(1000 \mathrm{~m}^{3}\right.$ par hectare et par an) pendant 5 années consécutives (19911995), les épandages ayant été arrêtés depuis lors. Un échantillonnage régulier des sols des différents horizons du profil (trois profondeurs) a été effectué depuis la mise en place du dispositif. En outre ce dispositif permet la collecte en continu des eaux de drainage de la parcelle traitée.

Ce dispositif constitue à ce titre un site observatoire privilégié et un modèle expérimental unique pour l'étude de l'impact des sur-épandages de lisier sur la qualité des sols et des eaux de drainage [5]. 
Les objectifs de cette étude consistent :

1) à quantifier dans le sol, les flux d'éléments (C, N, P, $\mathrm{K}, \mathrm{Cu}, \mathrm{Zn}$ ) apportés par les sur-épandages de lisier,

2) à suivre l'évolution de la composition chimique des eaux de drainage en distinguant la période d'accumulation des épandages intensifs (1991-1995) et la période de suivi des arrières-effets des épandages (1996-1999).

\section{MATÉRIEL ET MÉTHODES}

\subsection{Le dispositif expérimental}

En 1990, un pilote SOLEPUR grandeur réelle est mis en place sur une exploitation porcine à «Kéroignant " près de Plouvorn, dans le Finistère nord (29). Il comprend une parcelle aménagée $\left(3280 \mathrm{~m}^{2}\right)$ en drainage intégral qui reçoit des épandages intensifs et répétés de lisier de porc, un ensemble de bassins permettant la collecte, le stockage et le traitement des eaux de drainage issues de la parcelle et une parcelle non aménagée permettant in fine une filtration et l'épuration par le sol (fig. I).

Les parcelles épuratrices, finale et initiale, ainsi que la parcelle témoin sont cultivées avec du ray-grass anglais. Sur la parcelle épuratrice initiale, le couvert végétal de ray-grass était renouvelé à la fin de chaque saison d'épandages.

La parcelle épuratrice initiale et la parcelle témoin sont intégralement drainées. Un film polyane 15/100 étanche recouvre le fond et les bords des 2 parcelles. Les drains sont situés à $80 \mathrm{~cm}$ de profondeur. Ils sont recouverts de graviers et d'une géomembrane anti-colmatage.

Le substrat de la parcelle est constitué par les micaschistes de la Penzé. Il s'agit d'un sol brun faiblement lessivé à texture limon moyen sableux.

\subsection{Echantillonnage et analyses du lisier}

Lors de chaque épandage, un protocole de sous-échantillonnages multiples a été mis en place et systématiquement appliqué. Il consistait à suivre la rampe d'épandage lors de chaque passage de lisier, et à effectuer à intervalles de temps réguliers, des prélèvements au niveau de la buse-éclateur à l'aide d'un bécher de 2 litres. Ainsi, pour chaque passage de lisier, un échantillon de 30 à 60 litres de lisier était constitué. Par la suite, sur ce volume de lisier, un échantillon moyen était également constitué par une série de sous-échantillonnages, à des fins d'analyse.
Différentes analyses physico-chimiques sont effectuées sur chaque échantillon (52 échantillons analysés entre 1991 et 1995): matières sèches, matières organiques, carbone total, azote ammoniacal, azote total, phosphore total, potassium total, cuivre total, zinc total.

\subsection{Echantillonnage et analyses du sol}

Chaque année, au mois de mars, juste avant le premier épandage, des échantillons de terre ont été prélevés. Ils ont été réalisés comme suit: dans chaque parcelle T (témoin), et parcelle recevant les épandages (découpée à des fins statistiques en 4 blocs, A, B, C, D), neuf prélèvements sont effectués à l'aide d'une tarière manuelle. Les horizons $0-20$, 20-40 et $40-60 \mathrm{~cm}$ sont séparés. L'ensemble est tamisé sur place à $5 \mathrm{~mm}$. Un échantillon moyen de 1 à $2 \mathrm{~kg}$ est constitué puis mis en glacière pour être conservé et analysé. Une partie de cet échantillon est séché à $60^{\circ} \mathrm{C}$ puis broyé. Des analyses chimiques complètes sont réalisées sur ces échantillons : carbone total, azote total kjeldahl, phosphore extrait par la méthode Dyer, potassium échangeable, cuivre et zinc extrait à l'EDTA.

\subsection{Suivi et analyses des eaux de drainage}

Le site comporte un local technique. Divers appareils de mesures et de prélèvements y prennent place : (i) un préleveur automatique (ISCO 2100) d'échantillons d'eaux de drainage, en sortie de drains : 1 échantillon de $250 \mathrm{ml}$ toutes les 6 heures, soit 1 flacon de 1 litre par jour; (ii) un débitmètre (ISCO 3210) mesurant en continu, le débit (sonde ultrasons) et le volume cumulé drainé ; (iii) enfin un pluviomètre mesurant les précipitations en continu. Toutes ces informations sont enregistrées et stockées sur une centrale de mesure CR2M, avant d'être récupérées et traitées sur ordinateur (PC portable).

Un flacon de 11 d'eau de drainage est ainsi collecté à pas de temps journalier. Sur cet échantillon de 1 litre, une aliquote $(100 \mathrm{ml})$ est prélevée sur laquelle est réalisée une analyse quotidienne de la teneur en nitrates. Un échantillon composite est alors élaboré par le mélange de 7 à 10 échantillons journaliers sur lesquels une analyse chimique complète est réalisée.

Pour chaque échantillon quotidien, on effectue une analyse de la concentration en nitrates. On dispose ainsi du flux journalier d'azote lixivié. Pour chaque échantillon composite, une série d'analyses chimiques est effectuée comprenant

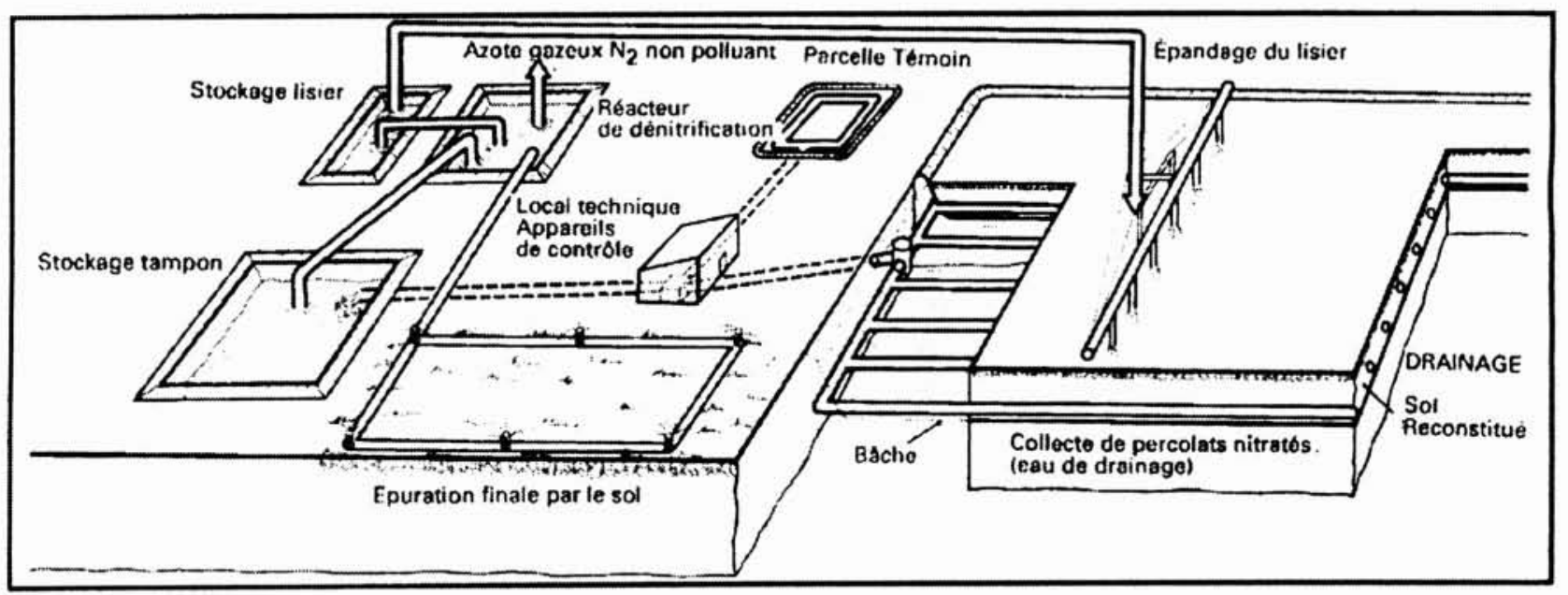

1. Description et fonctionnement du pilote Solepur. 
: potassium, calcium, ammonium, nitrite, nitrate, chlorures, sulfates, phosphates, phosphore total, azote total kjeldahl, demande chimique en oxygène (DCO), demande biochimique en oxygène (DBO). Pour quatre saisons de drainage consécutives, les échantillons d'eaux de drainage ont été également analysés pour leur teneur en éléments métalliques [2].

\section{III —RÉSULTATS}

\subsection{Les épandages de lisier sur la parcelle}

Trente épandages ont été réalisés entre 1991 et 1995 sur la parcelle épuratrice. Ces épandages ont été effectués sous couvert végétal, parfois après une coupe de ray-grass laissé sur le sol en " mulch». Pour l'une des cinq campagnes d'épandages (1994) ceux-ci ont été effectués sur sol nu, avec travail du sol après chaque épandage.

Ces cinq campagnes successives d'épandages massifs se sont déroulées sur les périodes comprises entre mars-avril et octobre-novembre de chaque année, soit un total cumulé de $4931 \mathrm{~m}^{3}$ de lisier par hectare $\left(986 \mathrm{~m}^{3} / \mathrm{ha} / \mathrm{an}\right)$. Au cours de ces 5 années, en moyenne 6 épandages par an ont été effectués apportant entre 58 et $245 \mathrm{~m}^{3}$ par hectare, soit un apport moyen pour les 30 épandages de l'ordre de $160 \mathrm{~m}^{3}$ de lisier par hectare et par épandage. Les épandages cumulés de lisier apportent près de 23 tonnes de carbone total par hectare et par an à la parcelle épuratrice, soit 115 tonnes de carbone par hectare apportées lors des 5 années d'épandage.

Les apports annuels d'azote à la parcelle épuratrice s'établissent à $4900 \mathrm{~kg} \mathrm{~N} / \mathrm{ha} / \mathrm{an}$, dont $2 / 3$ sous forme ammoniacale (soit un apport de l'ordre de $3200 \mathrm{~kg} \mathrm{~N}^{-\mathrm{NH}_{4}}{ }^{+} / \mathrm{ha} / \mathrm{an}$ ). Ainsi, l'azote total apporté entre 1991 et 1995 se situe à 24,5 tonnes d'azote, soit $800 \mathrm{~kg} \mathrm{~N} / \mathrm{ha}$ et par épandage. Les apports de phosphore correspondant, s'établissent en moyenne à $1600 \mathrm{~kg} \mathrm{P} / \mathrm{ha} / \mathrm{an}$, soit un apport cumulé de l'ordre de 8 tonnes de phosphore $(7964 \mathrm{~kg} \mathrm{P})$. Les épandages de lisier apportent également une charge importante en potassium, plus de $3300 \mathrm{~kg} \mathrm{~K} / \mathrm{ha} / \mathrm{an}$, soit un total cumulé sur la période 1991-1995 qui atteint 16,6 tonnes de potassium. Le calcium apporté s'établit à plus de $2400 \mathrm{~kg} \mathrm{Ca} / \mathrm{ha} / \mathrm{an}$, soit un total cumulé de plus de 12 tonnes de Ca.

Enfin, les épandages de lisier contiennent également des éléments trace ajoutés à l'alimentation, notamment cuivre et zinc. Ainsi, les apports de cuivre à la parcelle Solepur s'établissent à $37 \mathrm{~kg} \mathrm{Cu} / \mathrm{ha} / \mathrm{an}$, soit un total de plus de $180 \mathrm{~kg} \mathrm{Cu}$ apportés au sol. Les apports de zinc s'établissent à $53 \mathrm{~kg}$ $\mathrm{Zn} / \mathrm{ha} / \mathrm{an}$, soit un total cumulé de $266 \mathrm{~kg} \mathrm{Zn}$.

\section{- 3.2 Evolution physico-chimique du sol : observations générales}

Carbone et azote total : la teneur en carbone du sol augmente régulièrement sur l'horizon $0-20 \mathrm{~cm}$ de l'ordre de $20 \%$ par an pour passer de $1,82 \%$ en mars 1991 à $3,38 \%$ en mars 1996. Cette augmentation de la teneur en carbone total du sol s'accompagne donc d'un stockage total de carbone dans le sol qui s'établit à plus de 40 tonnes sur l'horizon $0-20 \mathrm{~cm}$ et de l'ordre de 10 tonnes sur l'horizon $20-40 \mathrm{~cm}$. Au total, le stock de carbone du sol sur le profil $0-60 \mathrm{~cm}$ passe de 102 à 153 tonnes de carbone, soit un taux de recouvrement apparent pour le carbone apporté par le lisier qui s'établit à $44 \%$. Le sol a ainsi dégradé sous forme de $\mathrm{CO}_{2}$ et d'eau, plus de 12 tonnes de carbone par an. Entre 1996 et 1999, soit au cours de la période dite de « dormance ", on observe une baisse du stock de carbone du sol de l'ordre de $20 \mathrm{t} / \mathrm{ha}$.
La teneur en azote total du sol de la parcelle Solepur passe de $0,176 \%$ en mars 1991 à $0,347 \%$ en mars 1996 , soit une augmentation moyenne de $20 \%$ par an pour l'horizon de surface $(0-20 \mathrm{~cm})$. La teneur en azote total de l'horizon inférieur $(20-40 \mathrm{~cm})$ passe de $0,139 \%$ à $0,181 \%$ alors qu'on note une légère augmentation de 0,071 à $0,092 \%$ pour l'horizon $40-60 \mathrm{~cm}$. Le stock d'azote du sol est ainsi doublé pour l'horizon de surface qui passe de $4576 \mathrm{~kg} \mathrm{~N} / \mathrm{ha}$ en mars 1991 à $9022 \mathrm{~kg} \mathrm{~N} / \mathrm{ha}$ en mars 1996. Au total le sol sur l'ensemble du profil étudié $(0-60 \mathrm{~cm})$ accumule plus de $6000 \mathrm{~kg} \mathrm{~N} / \mathrm{ha}$, soit $25 \%$ de l'azote total apporté sur la parcelle. Au cours de la période post-épandage, la teneur en azote total du sol de l'horizon $0-20 \mathrm{~cm}$, baisse et se situe à $0,28 \%$ en mars 1999 , soit un "déstockage " d'azote de l'ordre de $1700 \mathrm{~kg} \mathrm{~N} / \mathrm{ha}$ en 3 ans.

Phosphore-Dyer : la teneur en phosphore extractible au réactif Dyer (acide citrique à $2 \%$ ) augmente de façon importante puisque celle-ci est multipliée par trois, après seulement un an d'épandages. A la suite des cinq années consécutives d'épandages de lisier, la teneur en P-Dyer du sol est ainsi passée de $160 \mathrm{mg} \mathrm{P} / \mathrm{kg}$ de sol en mars 1991 à plus de $2200 \mathrm{mg} \mathrm{P} / \mathrm{kg}$ de sol en mars 1996, alors qu'au cours de la même période la teneur en P-Dyer de l'horizon $20-40 \mathrm{~cm}$ passait de moins de $100 \mathrm{mg} \mathrm{P} / \mathrm{kg}$ sol à $575 \mathrm{mg} \mathrm{P} / \mathrm{kg}$ de sol. Une légère augmentation était même détectée sur l'horizon profond qui passe de $22 \mathrm{mg} \mathrm{P} / \mathrm{kg}$ de sol à $36 \mathrm{mg} \mathrm{P} / \mathrm{kg}$ sol. Au total, sur la période étudiée (mars 1991 à mars 1996) le sol a accumulé près de $6600 \mathrm{~kg} \mathrm{P}$ Dyer/ ha soit un taux de recouvrement dans le sol du phosphore apporté par le lisier qui s'établit à $83 \%$ de l'apport. Pendant la période suivante (1996 à 1999) la teneur en PDyer de l'horizon 0-20 baisse, et se situe à moins de $1900 \mathrm{mg} \mathrm{P} / \mathrm{kg}$ de sol en mars 1999 alors que cette teneur augmente dans les horizons inférieurs, traduisant ainsi une migration de phosphore dans le profil.

Potassium échangeable : la teneur en potassium échangeable du sol augmente rapidement à la suite des épandages, puisque après un an d'épandages répétés, le stock de potassium échangeable, sur l'ensemble du profil est passé de moins de $2000 \mathrm{~kg} \mathrm{~K} / \mathrm{ha}$ à près de $4000 \mathrm{~kg} \mathrm{~K} / \mathrm{ha}$. Ainsi, sur l'horizon 0-20 cm, la teneur du sol passe de $340 \mathrm{mg} \mathrm{K} / \mathrm{kg}$ sol en mars 1991 à $1400 \mathrm{mg} \mathrm{K} / \mathrm{kg}$ sol en mars 1996 . Il en est de même pour l'horizon inférieur $(20-40 \mathrm{~cm})$ qui voit sa teneur en potassium échangeable passer de $240 \mathrm{mg} \mathrm{K} / \mathrm{kg}$ sol en mars 1991 à $1230 \mathrm{mg} \mathrm{K} / \mathrm{kg}$ sol en mars 1996. Enfin, l'horizon $40-60 \mathrm{~cm}$ voit également sa teneur en potassium augmenter fortement de $179 \mathrm{mg} \mathrm{K} / \mathrm{kg}$ sol en mars 1991 à plus de $860 \mathrm{mg} \mathrm{K} / \mathrm{kg}$ sol en mars 1996 à la fin des cinq campagnes d'épandages. On constate pour cet élément, contrairement à ce qui était observé pour le carbone, l'azote et le phosphore (où seuls les deux horizons labourés voyaient leur teneur augmentée), une migration importante du potassium qui «garnit » l'ensemble du profil de sol. Le stock de potassium du sol de la parcelle Solepur passe ainsi de $2000 \mathrm{~kg} \mathrm{~K} / \mathrm{ha}$ en mars 1991 à plus de $9000 \mathrm{~kg} \mathrm{~K} / \mathrm{ha}$ en mars 1996, soit un taux de recouvrement dans le sol par rapport au potassium épandu avec le lisier qui s'établit à $43 \%$. Durant la période de "dormance " du site, de mars 1996 à mars 1999, on observe une forte diminution de la teneur en potassium qui passe ainsi pour l'horizon supérieur $(0-20 \mathrm{~cm})$ de 1400 à $770 \mathrm{mg} \mathrm{K} / \mathrm{kg}$ de sol.

Cu et Zn EDTA : la teneur en cuivre extractible à l'EDTA, très faible dans le sol de départ en mars 1991, moins de $3 \mathrm{mg} / \mathrm{kg}$ sol pour l'horizon $0-20 \mathrm{~cm}$, augmente significativement dès la fin de la première campagne d'épandages pour se situer à près de $9 \mathrm{mg} / \mathrm{kg}$ sol. Pour l'horizon 20-40 la teneur en un an est doublée et passe de $2 \mathrm{mg} \mathrm{Cu}-$ EDTA $/ \mathrm{kg}$ sol en mars 1991 à plus de $4 \mathrm{mg} / \mathrm{kg}$ sol en mars 1992. Cette augmentation se poursuit consécutivement aux campagnes répétées de lisier pour atteindre plus de $35 \mathrm{mg}$ Cu-EDTA $/ \mathrm{kg}$ sol en mars 1996 pour l'horizon $0-20 \mathrm{~cm}$ et plus de $12 \mathrm{mg} \mathrm{Cu}$-EDTA/kg sol pour l'horizon $20-40 \mathrm{~cm}$. 
L'horizon inférieur $(40-60 \mathrm{~cm})$ voit sa teneur passer de $1,3 \mathrm{mg} / \mathrm{kg}$ à $2,2 \mathrm{mg} / \mathrm{kg}$. Pour la période considérée sur l'ensemble du profil $0-60 \mathrm{~cm}$, le stock de cuivre EDTA augmente ainsi de $16 \mathrm{~kg} \mathrm{Cu} / \mathrm{ha}$ en mars 1991 à près de $130 \mathrm{~kg} \mathrm{Cu} / \mathrm{ha}$ en mars 1996, soit un taux de recouvrement dans le sol qui se situe à $63 \%$.

Comme pour le cuivre les concentrations en zinc extractible à l'EDTA dans le sol augmentent de façon spectaculaire, et ce dès la fin de la première campagne d'épandages de lisier. Ainsi sur l'horizon 0-20 cm, la teneur du sol, faible au départ de l'essai, de l'ordre de $2-3 \mathrm{mg} \mathrm{Zn} / \mathrm{kg}$ sol (mars 1991) passe à $12-13 \mathrm{mg} / \mathrm{kg}$ sol dès mars 1992 . Le deuxième horizon échantillonné voit également sa teneur augmenter de $2 \mathrm{mg} / \mathrm{kg}$ à $5 \mathrm{mg} / \mathrm{kg}$. Au cours de la période considérée (mars 1991 à mars 1996) cette augmentation régulière des teneurs en zinc extractible à l'EDTA, conduit à un stock de zinc dans le sol qui passe de $15 \mathrm{~kg} \mathrm{Zn/ha} \mathrm{à} \mathrm{plus} \mathrm{de} 210 \mathrm{~kg} \mathrm{Zn} / \mathrm{ha}$, soit un pourcentage de recouvrement dans le sol qui s'établit à $74 \%$.

\section{- 3.3 Evolution chimique des eaux de drainage}

Drainage : le drainage annuel moyen sur les six dernières saisons allant de 1993/94 à 1998/99 s'établit à plus de $320 \mathrm{~mm}$, avec une saison particulièrement sèche (1996/97) et un drainage de $110 \mathrm{~mm}$, et une saison particulièrement pluvieuse (1994/95) avec près de $500 \mathrm{~mm}$ (fig. 2). Compte tenu de la superficie de la parcelle drainée Solepur $\left(3280 \mathrm{~m}^{2}\right)$, la lame d'eau moyenne recueillie dépasse les $1000 \mathrm{~m}^{3}$ d'eau

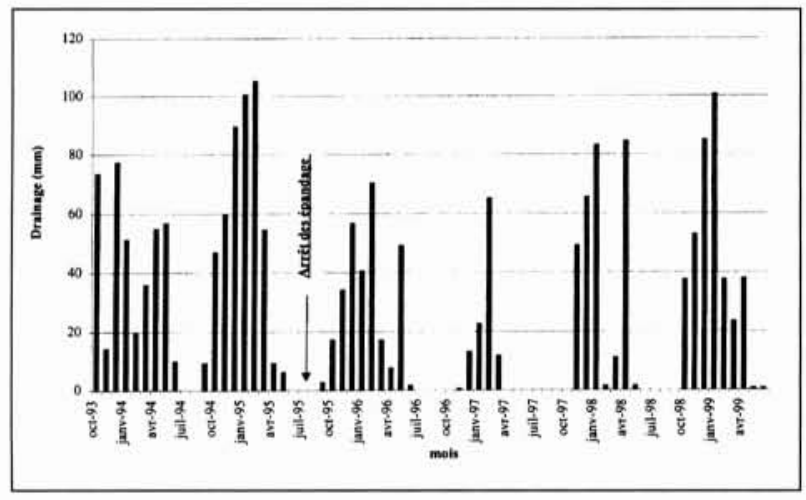

2. Drainage mensuel sous le lysimètre entre 1993-1999. par an. Au-delà de la quantité d'eau drainée annuellement, la deuxième observation qui s'impose en regard du dispositif Solepur est la limpidité de l'eau de drainage qui témoigne ainsi des capacités épuratrices, de filtration et de rétention du sol soumis à des épandages massifs de lisier brut chargé en matières en suspension et en matières sèches.

Flux de nitrates lixiviés : compte tenu des doses massives d'azote ammoniacal apporté annuellement entre 1991 et 1995 par les cinq campagnes sucessives d'épandages de

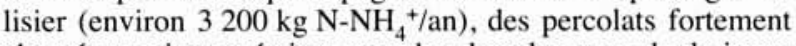
chargés en nitrates étaient attendus dans les eaux de drainage quittant la parcelle. Les concentrations en nitrates ont été établies en flux journalier et sont présentées en moyennes mensuelles (fig. 3) notamment pour les 6 dernières saisons de drainage. Ainsi, les concentrations moyennes en nitrates augmentent consécutivement à la succession des épandages de lisier (le dernier épandage a eu lieu le 31 octobre 1995) et atteignent plus de $400 \mathrm{mg} / \mathrm{l}$ d'azote nitrique lors de la saison de drainage 1995/96. Ce n'est que lors de la quatrième saison de drainage, après la fin des épandages de lisier soit $1998 / 99$, que la concentration moyenne en nitrates $\left(\mathrm{N}^{\left.-\mathrm{NO}_{3}{ }^{-}\right)}\right.$ commence à diminuer à moins de $50 \mathrm{mg} / \mathrm{l}$. Tous les échantillons d'eaux de drainage ont été analysés pour leur teneur en nitrites et ammonium. On ne détecte jamais de nitrites dans les eaux de drainage (moins de $0,1 \mathrm{mg} / \mathrm{l}$ ) ni d'azote ammoniacal (moins de $1 \mathrm{mg} / \mathrm{l}$ ).

Les flux de nitrates augmentent rapidement et consécutivement à l'accumulation des épandages. Sur la période considérée, plus de $3200 \mathrm{~kg}$ d'azote nitrique par hectare ont été lixiviés, soit 12 à $13 \%$ de l'azote total apporté par les épandages de lisier (24,5 t N/ha), et environ $20 \%$ de l'azote ammoniacal total apporté (environ $16 \mathrm{t} \mathrm{N}-\mathrm{NH}_{4}{ }^{+} / \mathrm{ha}$ ).

Lixiviation du potassium : comme nous avons pu le constater lors de l'analyse chimique du sol, le potassium apporté par les épandages répétés de lisier migre facilement dans le profil et atteint rapidement tous les horizons qui deviennent saturés. Il n'est donc pas surprenant de constater que les concentrations en potassium dans les eaux de drainage augmentent rapidement à l'issue des épandages et passent de $30 \mathrm{mg} / \mathrm{l}$ lors de la première saison de drainage (1991/92) à près de $300 \mathrm{mg} / \mathrm{l}$ lors de la saison de drainage 1995/96 (fig. 4). Le potassium lixivié, soit près de $3079 \mathrm{~kg} \mathrm{~K} / \mathrm{ha}$ représente ainsi $19 \%$ du potassium apporté par le lisier. Des pics de potassium à plus de $170 \mathrm{~kg} \mathrm{~K}$ lixiviés par hectare et par mois ont été observés à trois occasions : février 1996; janvier 1998 et avril 1998. Ainsi en dépit de l'arrêt des épandages en 1995, on observe un relargage important de cet élément avec 500 à $600 \mathrm{~kg} \mathrm{~K}$ lixiviés

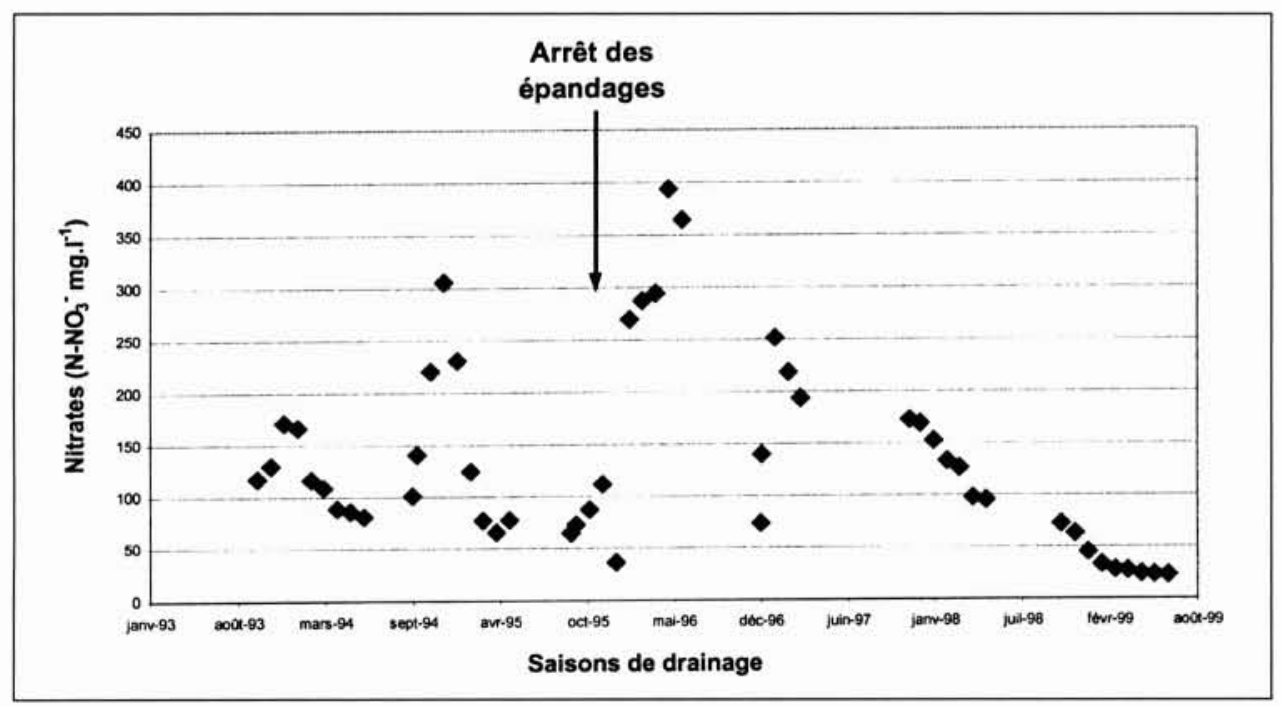

3. Evolution de la concentration moyenne mensuelle en azote nitrique lors des six dernières saisons de drainage (1993/94-1998/99). 
4. Evolution de la concentration moyenne mensuelle en potassium lors des six dernières saisons de drainage (1993/94-1998/99).
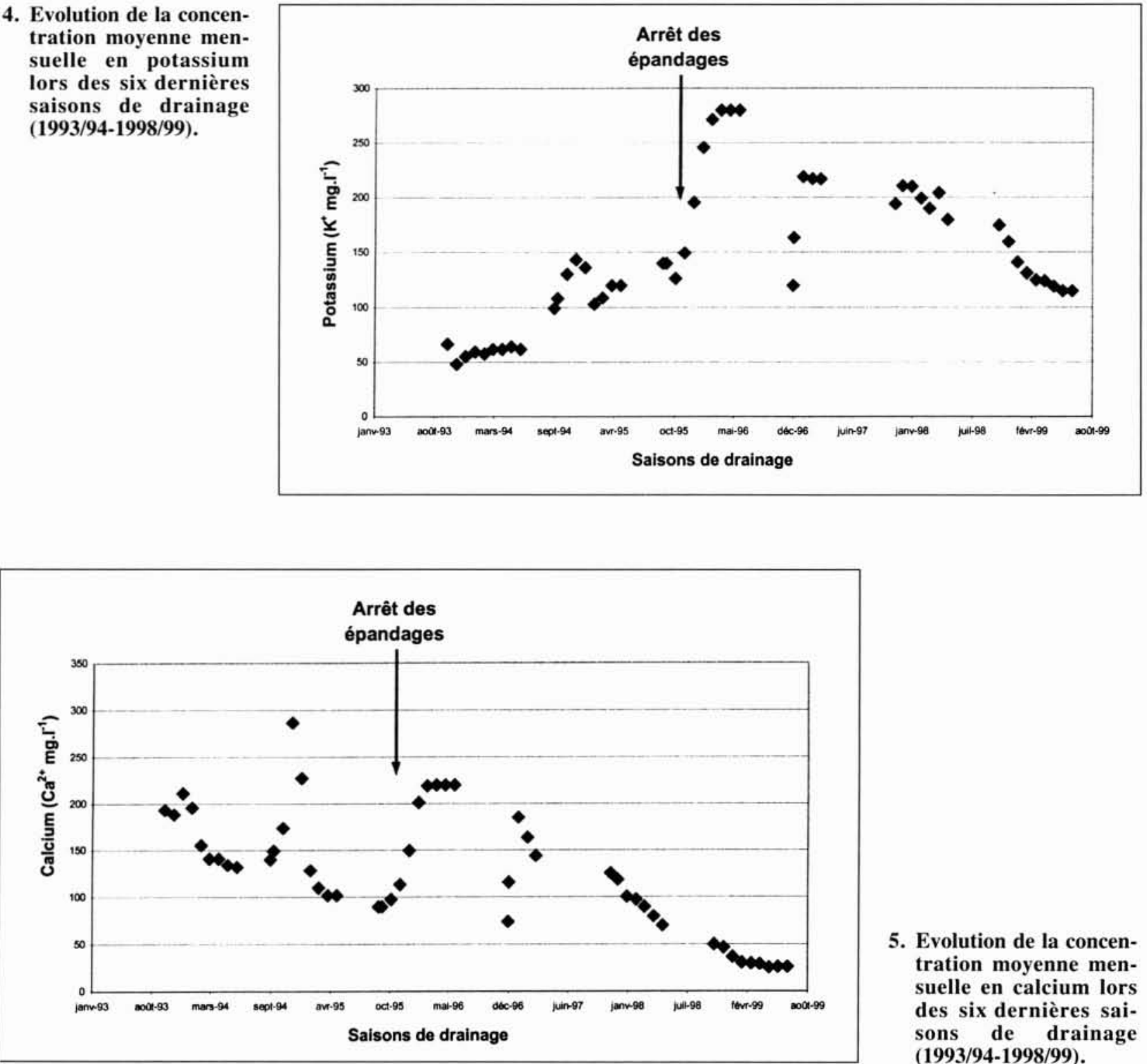

5. Evolution de la concentration moyenne mensuelle en calcium lors des six dernières saisons de drainage (1993/94-1998/99).

lors des deux dernières saisons de drainage 1997/98 et 1998/99.

Lixiviation du calcium : au cours des 5 dernières années de drainage, les flux de calcium $\left(\mathrm{Ca}^{++}\right)$s'établissent en moyenne à plus de $500 \mathrm{~kg} / \mathrm{ha} / \mathrm{an}$ soit environ $20 \%$ du calcium apporté par le lisier. La concentration moyenne en calcium dans les eaux, reste élevée lors des 4 saisons de drainage consécutives (allant de 1993/94 à 1996/97) et se situe entre 160 et $180 \mathrm{mg} / \mathrm{l}$. On observe une forte diminution de cette concentration à moins de $50 \mathrm{mg} / \mathrm{l}$ lors de la dernière saison de drainage (fig. 5).

Lixiviation des chlorures : la lixiviation des chlorures s'établit en moyenne sur les 5 dernières saisons de drainage à $600 \mathrm{~kg} \mathrm{Cl} / \mathrm{ha} / \mathrm{an}$, soit plus de 3 tonnes de chlorures lixiviés. La concentration moyenne en chlorures, par saison de drainage, s'établit respectivement à 237, 160,243, 256 et $106 \mathrm{mg} / \mathrm{l}$, pour les saisons allant de 1993/94 à 1997/98. Lors de la saison consécutive à la dernière campagne d'épandages intensifs (soit 1995/96), des pics de concentration en chlorures à plus de $350 \mathrm{mg} / \mathrm{l}$ avaient été observés (fig. 6). La tendance, lors de la saison de drainage en cours (1998/99), est la baisse de cette concentration à moins de $30 \mathrm{mg} / \mathrm{l}$, proche de celle observée sur la parcelle témoin.
Lixiviation des sulfates : les quantités moyennes de sulfates $\left(\mathrm{SO}_{4}{ }^{2-}\right)$ lixiviés sont proches de celles des chlorures et s'établissent en moyenne, au cours des 5 dernières saisons de drainage, à $580 \mathrm{~kg} \mathrm{SO}_{4}{ }^{2-} / \mathrm{ha} / \mathrm{an}$, soit de l'ordre de 3 tonnes de sulfates lixiviés. Par contre, la concentration moyenne dans les eaux de drainage demeure élevée et s'établit à $200 \mathrm{mg} / \mathrm{l}, \mathrm{y}$ compris pour la saison de drainage actuelle.

Autres éléments (phosphore, métaux) : les concentrations en phosphore total dans les eaux de drainage ont été systématiquement mesurées. Elles s'établissent régulièrement à moins de $10-20 \mu \mathrm{g} / \mathrm{l}$ de P. Compte tenu des quantités massives de phosphore apporté par le lisier (environ $8000 \mathrm{~kg} \mathrm{P} /$ ha sur 5 ans), ce flux de phosphore dans l'eau est négligeable et représente au total sur les 5 ans, moins de $300 \mathrm{~g}$ de $\mathrm{P}$ lixivié (soit $0,003 \%$ de l'apport). Les concentrations en métaux trace ont été déterminées régulièrement lors de 4 saisons de drainage. Pour le cuivre, les concentrations observées dans les eaux s'établissent entre 1 et $6 \mu \mathrm{g} / 1$, soit un flux maximum cumulé sur l'ensemble de la période de drainage qui s'élève à $100 \mathrm{~g} \mathrm{Cu}$ lixiviés (pourcentage de recouvrement par rapport au cuivre apporté par le lisier de l'ordre de $0,05 \%$ ). Pour le zinc, les teneurs dans les eaux de drainage s'établissent à moins de $100 \mu \mathrm{g} / \mathrm{l}$, soit un flux 


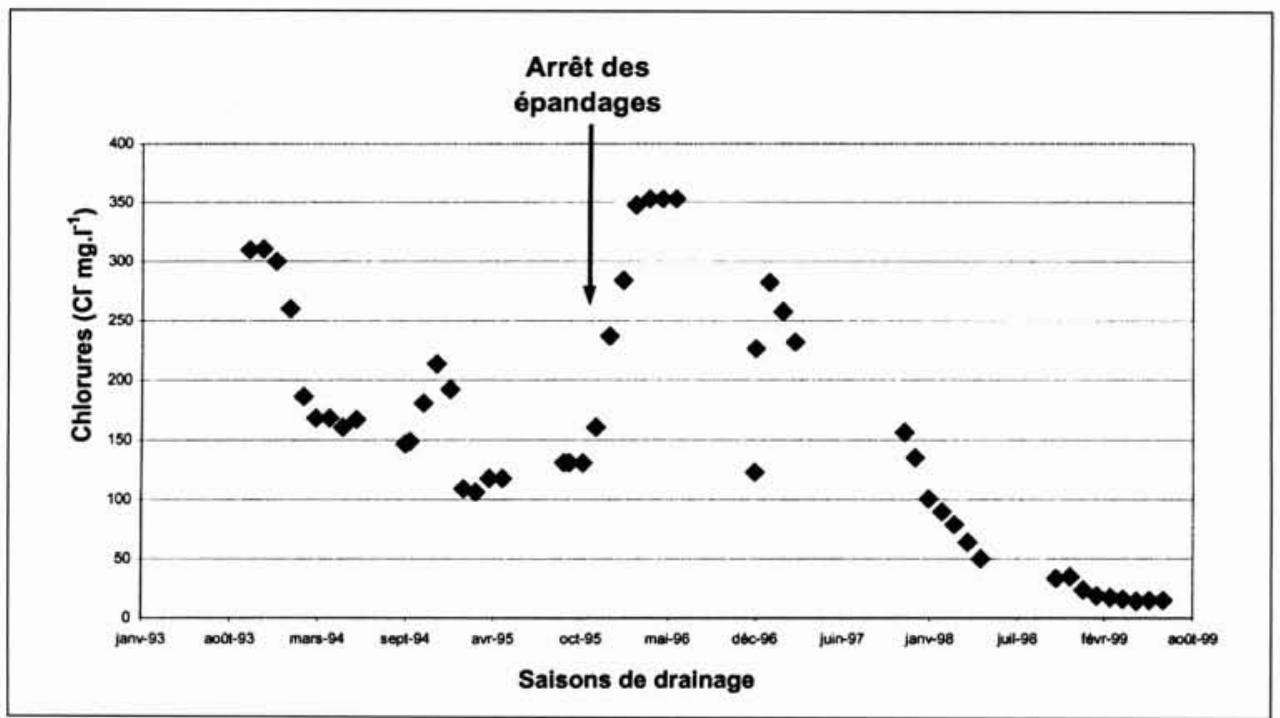

6. Evolution de la concentration moyenne mensuelle en chlorures lors des six dernières saisons de drainage (1993/94-1998/99).

maximum cumulé de l'ordre de $1660 \mathrm{~g} \mathrm{Zn}$. Le pourcentage de recouvrement, par rapport au zinc apporté par le lisier, s'établit ainsi à moins de $0,6 \%$.

\section{IV $\square$ CONCLUSION}

L'expérience acquise sur le dispositif en vraie grandeur Solepur démontre que le sol est un système aux propriétés épuratrices indéniables, mais dont la capacité peut être aussi saturée. Au-delà des éléments carbone et azote qui sont fortement minéralisés, les autres éléments apportés par le lisier sont, soit retenus sur le filtre sol (Phosphore, métaux lourds $\mathrm{Cu}, \mathrm{Zn}$ ), soit directement entraînés vers les eaux de drainage (potassium, calcium, chlorures, sulfates etc).

Ces capacités épuratrices du sol, sont donc aussi limitées par des seuils de concentration en différents éléments nuisibles ou toxiques qui peuvent passer en solution dans les eaux. Notre étude a ainsi permis d'apporter un éclairage global sur ces différents risques d'accumulation et de transfert de nutriments.

\section{BIBLIOGRAPHIE}

[1] Germon JC (1985). Le sol, un système épurateur efficace...s'il est bien géré. Revue du Palais de la Découverte, vol. $14, \mathrm{n}^{\circ} 133,19-41$

[2] L'Herroux L, Le Roux S, Appriou P et Martinez J (1997) Behaviour of metals following intensive pig slurry applications to a natural field treatment process in Brittany (France). Environmental Pollution 97, 119-130.

[3] Maresca B (1979). L'épandage des eaux usées. Manuel de recommandations techniques. La Documentation Française. Paris.

[4] Martinez J (1997) Solepur : a soil treatment process for pig slurry with subsequent denitrification of drainage water. Journal of Agricultural Engineering Research 66, 51-62.

[5] Martinez J et Peu P (1999). Etude de suivi d'un dispositif expérimental (biologique et physico-chimique) de sur-épandage de lisiers de porcs $\left(1000 \mathrm{~m}^{3} / \mathrm{ha} / \mathrm{an}\right)$. Rapport Etude Agence de I'Eau Loire Bretagne-Convention n`7971640. 23 p. + annexes. 